\title{
Paraplegia from Schistosomiasis
}

\author{
K. Bloom, MD, ${ }^{1}$ M. M. Freed, $\mathbf{M D}^{2}$ \\ ${ }^{1}$ Staff Psychiatrist, Moss Rehabilitation Hospital, ${ }^{2}$ Chief of Rehabilitation Medicine, \\ Department of Rehabilitation Medicine, University Hospital of Boston Medical Center, \\ Boston, MA, USA.
}

\section{Summary}

Paraplegia, a rare complication of schistosomiasis has been reported in the literature. However, a detailed description of neurological recovery and long-term functional followup has not been published. In the cases presented, one patient experienced slow recovery over several years and remains nonambulatory. The other patient, infected at the same time and place, has completely recovered neurologically. Recovery was independent of the rehabilitation efforts applied. Case reports, discussion of spinal cord injury due to schistosomiasis and recommendations for treatment are presented in detail.

Key words: Spinal cord diseases, Schistosomiasis, Paraplegia, Parasitic diseases.

Note: These cases were reported in part in Morbidity and Mortality Weekly Report ${ }^{6}$ and case 1 was reported in part in the clinicopathological exercise of the New England Journal of Medicine. ${ }^{2}$

Schistosomiasis, a human blood fluke infection, is endemic in Africa, South America, and Asia. Infection with one of the three types of blood flukesSchistosoma mansoni, Schistosoma japonicum and Schistosoma haematobium-may cause a severe degree of morbidity, and left undiagnosed may result in major disability and mortality. ${ }^{10}$

In the United States, infection with human schistosomes has been estimated to exceed 400000 persons. ${ }^{16}$ These are mainly in people who are immigrants from endemic areas, in particular Puerto Rico, Brazil, the Middle East, and the Philippines. ${ }^{10}$ The infection cannot be transmitted in the United States because of the absence of the appropriate snail intermediate host.

The life cycle of the 3 schistosome species requires man as the definitive host. The adult worms (1-2 cm in length) live in the venous system of the intestines or urinary bladder. After mating, they pass eggs which penetrate into the lumen of the intestines urinary bladder and are then excreted in the host's faeces or urine.

Reprint requests to: Dr M. M. Freed, MD, Chief, Department of Rehabilitation Medicine, University Hospital, 88 East Newton Street, Boston, MA 02118, USA

Supported in part by RSA Grant no. 572419178981-6 and NIORR Grant no. T657770720 
The eggs hatch in fresh water and release ciliated, motile miracidia that penetrate into the body of the snail intermediate host and multiply. In 4 to 6 weeks, hundreds of motile forked tail cercariae emerge from the snail. On encountering human skin, these infective forms penetrate, lose their tails, and become schistosomula. The schistosomula migrate to the lungs and liver and in approximately 6 weeks they mature into adult worms that mate and migrate via the venous system to their final habitat. ${ }^{10} S$. mansoni mainly live in venules adjacent to the colon, $S$. japonicum reside in the venules of the small intestine, and $S$. haematobium are found in the venules of the urinary bladder. Some of the eggs do not pass through the wall of the intestines or urinary bladder but are swept by the venous stream and lodge in various tissues where they degenerate and form granulomas.

Although over 200000 million people world-wide ${ }^{10}$ are infected with schistosoma; there have been only 64 cases reported with involvement of the spinal cord with at least 41 of these caused by $S$. mansoni ( 8 were not specified as to the type of schistosome). ${ }^{15}$ Diagnosis is best made by examination for ova in the stool $(S$. mansoni and $S$. japonicum) or urine (S. haematobium) as well as a detailed neurological examination.

\section{Case report 1}

A 21-year-old healthy white male who had never travelled outside the United States was fully immunized against tetanus, typhoid, cholera, and yellow fever and received an injection of gamma globulin prior to his departure for Kenya in February, 1984. While in Kenya, he took chloroquine, sulfadoxine and pyrimethamine (Fansidar) for malaria prophylaxis. In early March, he swam in a small body of water he and his colleagues had dammed off from a small stream. In early April he complained of fever, abdominal pain, and diarrhoea without blood or mucus. This resolved with no treatment. On April 26, he became ill again. This time he complained of fever, chills, sweats, anorexia, mild non-bloody diarrhoea, and abdominal pain. He was treated orally for presumptive malaria. His symptoms abated. However, on April 28, he developed severe lumbar back pain without tenderness or radiation. He had associated numbness in both feet. There was no weakness. On May 1 he developed difficulty with proprioception and pronounced weakness in the lower extremities. On May 2 he became ataxic and developed urinary retention. Stool examination showed many ova of $S$. mansoni. A diagnosis of $S$. mansoni infection was made, and he was treated with praziquantel $40 \mathrm{mg} / \mathrm{kg}$ in a single dose and prednisone $20 \mathrm{mg}$ every 8 hours. The steroids were tapered after 2 months. Tapering was continued over 5 months. The patient was transported to the United States on May 5. His neurological examination revealed T11 Frankel A paraplegia. ${ }^{8}$ There were no superficial or deep tendon reflexes below T11. There was no rash, fever, lymphadenopathy, hepatosplenomegaly or tenderness on percussion of the spine. There was no block or mass lesion seen on a myelogram. CT scan showed the lumbar cord to be slightly enlarged with no focal abnormalities. His prednisone was increased to $20 \mathrm{mg}$ every 6 hours.

On May 15, the patient was transferred to the New England Spinal Cord Injury Center of University Hospital in Boston. His neurological findings had not changed. By May 24, he had developed Grade 2-strength bilaterally in most of his hip and knee muscles. The muscle grades were symmetrical. At the end of a 3 month admission, he had mostly grade 2 to 3 strength in most of his lower extremity muscles including his ankles, feet, and toes. Once again, the grades of strength in the muscles were relatively symmetrical.

During his 3 months of rehabilitation, the patient received intensive occupational and physical therapy. In therapy, the patient strengthened unaffected and weak muscles. He learned to manage a wheelchair, transfer himself from wheelchair to bed and commode as well as to dress and bathe himself. He regained muscle activity in his lower extremities and began ambulating with knee-ankle-foot-orthoses and a walker. On discharge, he was a T12 
Frankel D paraplegic. Since he had no spontaneous voiding, he managed his bladder with intermittent catheterisation.

Two years later, the patient's level continues to descend. He continues to have absent proprioception and deep tendon reflexes in his lower extremities. He has hyperesthaesia in both lower extremities, and has a sensation of bladder fullness and knows when he is emptying. He reinforces bladder emptying by the Crède manoeuver. He achieves erection but not ejaculation. He is a L4 Frankel $\mathrm{D}$ as calculated by his score on the motor index. ${ }^{9}$ He ambulates $1 \frac{1}{4}$ hours a day with bilateral ankle foot orthoses with a walker. He would walk for longer periods of time but does not because he is troubled by the lack of proprioception in his lower extremities.

\section{Case report 2}

A 20-year-old white female also travelled to Kenya. She was also fully immunized against tetanus, typhoid, cholera, and yellow fever and took chloroquine sulfadoxine and pyrimethamine (Fansidar) for malaria prophylaxis. On April 29, 1984 she developed fever, abdominal pain, and non-bloody diarrhoea. She was treated with ampicillin for gram positive cocci in her urine and with metronidazole. On May 3, she developed severe back pain without radiation, weakness, or bladder symptoms. From May 7 to May 9, she rapidly became non-ambulatory because of lower extremity weakness. She also complained of difficulty starting her stream of urine. On May 9, stool examination showed $S$. mansoni. She was treated with oxamniquine $15 \mathrm{mg} / \mathrm{kg}$ in split dosage and prednisone $40 \mathrm{mg}$ initially followed by $20 \mathrm{mg} 3$ times a day for $S$. mansoni transverse myelitis. On arrival in the United States on May 11, her neurological examination showed Ll-2 flaccid paraplegia. Vibratory sense was intact but sensation to touch and pinpoint were absent below the level. There were no reflexes in the lower extremities.

A myelogram showed no obstruction, but a CT scan showed some swelling of the conus medullaris. Stool examination showed many $S$. mansoni ova. Because the dose of oxamniquine was considered inadequate, the patient was treated with praziquantel $20 \mathrm{mg} / \mathrm{kg}$ every 8 hours for 3 doses. She also received dexamethasone $10 \mathrm{mg} 4$ times a day. Within 4-6 hours of starting the dexamethasone, her severe back pain improved. By the second day of treatment, the patient's motor function and sensation had improved, although the deep tendon reflexes were still absent. The patient was continued on steroids for an additional month and a half. On May 15, she began moving both lower extremities against gravity. On May 25, the patient was admitted to the Rehabilitation Institute of Chicago for comprehensive rehabilitation. Her comprehensive rehabilitation program consisted of physical and occupational therapy. She strengthened unaffected and weak muscles and learned to perform activities of daily living with her paretic lower extremities. She also was given gait training with assistive devices. By June 6 she had descended to L1 Frankel D right and S1 Frankel D left. She has subsequently descended to L1 Frankel E right and S1 Frankel E left. She continues to have some bladder urgency and stress incontinence. She has normal tone in her lower extremities and ambulates without orthoses or other assistive devices.

\section{Discussion}

Clinical involvement of the spinal cord in schistosomiasis is rare. When it occurs, schistosomiasis most commonly involves the low thoracic and high lumbar regions. This is thought to be due to the free anastomoses between the pelvic veins and the valveless vertebral plexus. ${ }^{13}$ Others have suggested that the ova reach the cord by arterial embolisation through the 'arteria radiculus magna anterior' as described by Adamkiewicz in 1881. This artery is the largest anterior feeder located in the upper lumbar region of the cord. It occurs unilaterally and is usually on the left side. ${ }^{4,5}$ Still another possibility is that the spinal cord involvement stems from an 
immuni-allergic reaction from products derived from dead worms and their eggs. ${ }^{12}$

Infection of the spinal cord with schistosomes can be divided into 4 pathological types. ${ }^{4}$ The myelitic type occurs when the degree of tissue reaction to the infection is minimal. ${ }^{5}$ The lesion consists of little reaction around the ova with destruction, vacuolation and atrophy of the cord. ${ }^{4}$ The granulomatous type occurs when the degree of reaction is intense. ${ }^{5}$ The lesion is formed as a result of intense gliotic and fibrotic reaction to the ova. ${ }^{4}$ The radicular type presents as a multi-root syndrome resulting from the presence of multiple granulomas in spinal roots. ${ }^{1}$ Anterior spinal artery occlusion is postulated to occur secondary to an arteritis which leads to obstruction of the anterior spinal artery or one of its tributary anterior radicular branches. ${ }^{14}$

Recommendations for treatment of spinal cord involvement vary. Most believe, in the older literature that a decompressive laminectomy with wide opening of the dura and a biopsy followed by antischistosomal agents was the regimen of choice. However, with praziquantel, it is possible that the need for surgery may be obviated. However, where there is a complete block on a myelogram, immediate decompressive laminectomy together with freeing of the cauda roots and biopsy is suggested. ${ }^{4}$ In cases of transverse myelitis and early granulomas, medical treatment is probably sufficient. ${ }^{1,4,12}$ All recommendations include the use of antischistosomal agents such as praziquantel or oxamniquine with or without corticosteroids. In some reports, ${ }^{7,11,15}$ there is evidence that early treatment with appropriate antischistosomal agents will produce recovery. It is therefore important to make a prompt diagnosis and begin treatment early. ${ }^{3}$ Patients with complete neurological recovery would not require a rehabilitation program. However, patients with partial or no recovery require participation in a comprehensive rehabilitation program.

The 2 patients discussed had transverse-myelitic types of $S$. mansoni and therefore were not treated surgically. Swelling of the involved areas on CT scanning was found although there was no block on myelogram. Both patients were treated with praziquantel and corticosteroids. The 2 patients participated in comprehensive rehabilitation programs which were designed to make them independent and functional with their disabilities. The programs were adapted as they improved.

\section{References}

1 Bird AV. Spinal cord complications of bilharziasis. S Afr Med J 1965: 39:158-162.

2 Case records of the Massachusetts General Hospital: Weekly clinicopathological exercises. N Engl J Med 1985: 312:1376-1383.

3 Cosnet JE, Van Dellen JR. Schistosomiasis (bilharzia) of the spinal cord: case reports and clinical profile. Q J Med 1986: 61:1131-1139.

4 El-Banhawy A. Schistosomiasis of the spinal cord, conus and cauda. Neurol Med Chir 1971: $11: 17-33$.

5 El-Banhawy A, Elwan O, Taher Y. Bilharzial granuloma of the conus medullaris and cauda equina. Paraplegia 1972: 10:172-180.

6 Epidemiologic Notes and Reports: Acute schistosomiasis with transverse myelitis in American students returning from Kenya. MMWR 1984: 33:445-447.

7 Efthimiou J, Denning D. Spinal cord disease due to Schistosoma mansoni successfully treated with oxamniquine. Bri Med J 1984: 288:1343-1344.

8 Frankel HL, Hancock DO, Hyslop G, et al. Value of postural reduction in the initial management of closed injuries of the spine with paraplegia and tetraplegia. Paraplegia 1969: 7:179-192.

9 Lucas JT, Drucker TB. Motor classification of spinal injuries with mobility, morbidity, and recovery indices. Am Surg 1979: 45:151-158. 
10 Mahmoud AAF Trematodes (Schistosomiasis, other flukes). In: Mandell GL, Douglas RG, Bennett JE eds. Principles and practice of infectious diseases. John Wiley, New York 1985: pp 15731579.

11 Molyneux ME, Galatius-Jensen F. Successful drug treatment of schistosomal myelopathy: a case report. S Afr Med J 1978: 54:871-872.

12 Neves J, Marinho RP, DeAraujo PK, Raso P. Spinal cord complications of the acute Schistosomiasis mansoni. Trans R Soc of Trop Med Hyg 1973: 67:782-792.

13 Pool GM. Tropical paraplegia: A case of transverse myelitis probably caused by Schistosomiasis mansoni. Paraplegia 1978: 16:113-117.

14 Siddorn JA. Schistosomiasis and anterior spinal artery occlusion. Am J Trop Med Hyg 1978: 27: 532-534.

15 Suchet I, Klein C, Horwitz T, Lalla S, Doodha M. Spinal cord schistosomiasis: a case report and review of the literature. Paraplegia 1987: 25:491-496.

16 Warren KS. Helminthic diseases endemic in the United States. Am J Trop Med Hyg 1974: 23: 723-730. 